Eine Gemeinsamkeit von so verschiedenen Organisationen, wie der Bürgerwehr von Graz und dem collectif anticrack des XIX. Bezirks von Paris, ist der schwindende Glaube an den Staat und das nachlassende Vertrauen in seine Schutzmacht. Das ist natürlich eine sensible Wahrnehmung, obwohl sie bei den hier zitierten sozialen Bewegungen auf ein sehr begrenztes Gebiet beschränkt bleibt: Die Instrumente des Nationalstaates waren nie dazu gedacht gewesen, globale Probleme zu bewältigen. Der Nationalstaat ist zunehmend hilflos, wenn die Regulierung internationaler Märkte - seien sie nun legal oder illegal - ansteht.

Wenn angesichts »der Globalisierung « die Ordnungsmacht des Nationalstaates abnimmt und er »schwach « wird, entsteht das Bedürfnis, die Verteidigung auf anderen Ebenen auszubauen und andere Ordnungs- und Schutzmächte zu entwickeln. Gut bekannt sind Organisationen, die auf der Ähnlichkeit der Interessen, Problemlagen und Lebensstile ihrer Mitglieder aufbauen, wie zum Beispiel Vereine von Eltern drogenabhängiger Kinder oder in manchen Ländern auch Vereine von Drogenabhängigen selbst. Im Gegensatz zu ihnen sind die oben beschriebenen Organisationen auf Gemeinsamkeiten aufgebaut, die fast vergessen schienen: Nachbarschaft, räumliche Nähe und teils auch Sprache und Ethnizität. Aber in einer Welt, die unübersichtlich geworden ist, erscheint das eigene Lebensumfeld wohl einerseits als gefährdeter, aber andererseits auch als wertvoller und der Verteidigung würdig.

Die Schutzmächte, die unterhalb der staatlichen Ebene aufgebaut werden, gehen ganz offensichtlich mit einem geringeren Grad an Neutralität gegenüber dem "zu Kontrollierenden « einher und sind von offen feindseligen Haltungen gegen »den Feind « getragen. Staatliche Maßnahmen sind üblicherweise streng geregelt, sie werden von Experten angeleitet und überwacht - bei den zivilen Schutzmächten ist dies in geringerem Maße bis gar nicht der Fall. Der Aufbau und die Ausübung ziviler Verteidigung erlaubt es daher emotionaler zu sein, und dies dürfte wiederum viele anziehen. In Abhängigkeit davon, welche politische Farbe die zivile Schutzmacht und ihre Mitglieder tragen, und auch in Abhängigkeit von den politischen Traditionen in die sie eingebettet sind, ist der Feind einmal ein "facho" und einmal ein schwarzafrikanischer Flüchtling. Aber in jedem Fall scheint der Feind aus sozialen Lagen zu stammen, die jenen, die ihr Lebensumfeld verteidigen, sehr unvertraut sind. Er oder sie ist »das Andere«, das von der Gemeinschaft auszuschließen ist.

Der Aufbau ziviler Schutzorganisationen gegen den Drogenhandel ist auch im Kontext der Risikoprävention zu betrachten, wie sie in industrialisierten Ländern zur Zeit vorwiegend eingesetzt wird. In dieser Form zielt sie auf die Differenzierung von Wahrnehmung, Empfindung und Erleben, wie auf die Steigerung von Aktivität, sie zielt auf die "Ermächtigung « von Einzelnen und sozialen Aggregaten, wie unter anderem von Gemeinden und Organisationen - oft auf Kosten des Verstehens von umfassenderen sozialen Prozessen und politischen Zusammenhängen. Güter, die bisher einen öffentlichen Charakter hatten, wie Gesundheit und Sicherheit, werden in diesem »Verfahren « zu Gütern, die (individuell) hergestellt und gekauft werden können - von Einzelnen, oder doch zumindest von der Gemeinde und den Organisationen, denen man angehört. Dies entspricht teilweise der Realität, bildet sie aber keineswegs zur Gänze ab: die komplexen "globalen « Interdependenzen werden in diesem Verfahren vernachlässigt, wenn sie nicht sogar ausgeschlossen werden müssen.

Die zivile Verteidigung gegen die Drogen kann schließlich auch im Kontext der schwierigen Bildung von Identität betrachtet werden. In einer Konsumgesellschaft muss Identität notgedrungen ein »Konsumentenprofil « umfassen, wenn es nicht sogar eines solchen als Basis bedarf. Ein Werbespruch bringt es auf den Punkt. »Ich konsumiere, also bin ich «. Ein anderer leitet folgendermaßen zum Fremd- und Selbstverständnis an: »Sage mir was Du isst und trinkst, und ich sage Dir, wer Du bist «. Auch die Ablehnung von Konsumgütern informiert darüber, wer man ist. Vermutlich eignen sich die Haltungen gegenüber psychoaktiven Substanzen besonders für die Bildung und Darstellung von Identität. Ein Weintrinker ist anders als ein Biertrinker, ein Marihuanagebraucher anders als einer der Heroin injiziert und einer, der seinen Bezirk gegen Dealer verteidigt, unterscheidet sich von allen anderen. Haltungen zu psychoaktiven Substanzen erlauben es zunehmend auch den politischen Parteien, sich zu differenzieren und ein mehr oder minder scharfes, unterschiedliches Profil zu entwickeln: wie zum Beispiel der FPÖ, die, wie bereits angemerkt, auf eine drogenfreie Gesellschaft setzt.

Aber vielleicht ist auch alles viel einfacher, vielleicht geht es nur darum einen Fuß auf den Boden dieser chaotischen und zunehmend unübersichtlichen Welt zu bekommen, wenn man gegen etwas kämpft, das man als »unserer Lebensweise « nicht zugehörig erlebt. »Our way of life - OWL « hat es Patricia Highsmith in ihrem berühmten Roman «Das Zittern des Fälschers« genannt. Wenn diese Annahme zutrifft, haben wir in der nahem Zukunft mehr und mehr der Erscheinungen $\mathrm{zu}$ erwarten, die oben beschrieben wurden.

Irmgard Eisenbach-Stangl forscht am Ludwig-Boltzmann-Institut für Suchtforschung in Wien ettina Paul:

\section{»Do not disturb!« \\ Der erfolgreiche Transfer privaten Protestes in politische Programme}

Die Entwicklung privater Initiativen, wie sie Irmgard Eisenbach-Stangl für Österreich beschreibt, sind auch in Deutschland ein bekanntes, sogar alltägliches Phänomen. Doch gibt es wesentliche Unterschiede.

Der Zusammenschluss von Bürger/innen zur Erhöhung eines »Sicherheitsgefühls« im eigenen Wohnumfeld wird mit einer diffusen Kriminalitätsbedrohung und dem Wunsch nach Schutz für ihr Eigentum und die körperliche Unversehrtheit ihrer Familien begründet. Diese so genannte »Kriminalitätsfurcht « vereint diverse Ängste, Befürchtungen, Aversionen u.Ä. zu einem Bedrohungsmoment, egal, ob sich die Abneigung dabei gegen den Zustand der Dunkelheit, gegen Personen, unbekannte Lebensstile oder medial-vermittelte Szenarien richtet. Die Tätigkeiten privater Initiativen zeugen von dieser Bedrohungskonstruktion. Im Jahre 2000 wurde in Rahlstedt, einem weitläufigen Stadtteil am Rande Hamburgs, eine Initiative von 40 Bewohner/innen ins Leben gerufen, die sich »Mehr Sicherheit für Rahlstedt e.V.« nennt. Ihre Aktivitäten umfassen abendliche Patrouillen in ihrer (gutbürgerlichen) Nachbarschaft. Ausgerüstet mit Taschenlampen und Handys suchen sie nach verdächtigen Personen oder Begebenheiten. Jede Person, die der Nachbarschaft nicht bekannt ist, steht erst einmal unter Verdacht, vor allem aber solche, bei denen die äußerliche Erscheinung als Gefahrenindiz gewertet wird. Diese Erscheinungen beinhalten für die Gegend unübliche Anzeichen von Armut, Jugendkultur oder kultureller Herkunft. Der Fremdheitsaspekt wirkt bedrohlich, wird mit einem Angriff auf die Gemeinschaft gleichgesetzt. Die Initiative versteht sich dabei als Ergänzung zur Polizei, nicht als deren Ersatz. Als Grund für die Aufnahme ihrer Aktivitäten führt sie an, dass die staatlichen Einsatzkräfte nicht in der Lage seien, sie und ihre Familien ausreichend zu beschützen.

Phänomene, wie die der Rahlstedter Initiative, sind in Deutschland nicht selten, trotzdem hat sich parallel dazu eine weitreichende kommerzielle Sicherheitsindustrie entwickelt, die die meisten privaten Aktivitäten auf diesem Gebiet übernommen hat. Dies ist ebenfalls in diesem Teil Hamburgs zu beobachten. Eine private Sicherheitsfirma fährt dort ebenfalls ihre Patrouillen. Während die verbliebenen privaten Initiativen hauptsächlich am Rande der großen Städte aktiv sind, in 
wohlhabenden Gegenden oder zumindest, wie in Rahlstedt, in solchen mit vielen Einfamilienhäusern, erstreckt sich das Tätigkeitsgebiet der Sicherheitsfirmen über das gesamte Stadtgebiet. Unter den von den privaten Initiativen abzuwendenden Bedrohungen kommt der Drogenthematik kein besonderer Stellenwert zu, wahrscheinlich, weil die öffentliche Sichtbarkeit von Drogenkonsum oder Obdachlosigkeit hauptsächlich im Zentrum der Stadt (oder der Stadtteile) Platz einnimmt, und daher eben gerade nicht an den Orten, an denen die Bewohner/innen die Forderung nach der Patrouillierung ihrer Nachbarschaft erheben.

\section{Private Initiativen gegen öffentlich sicht- baren Drogenkonsum und - Handel - ein etwas anderes Phänomen}

In Hamburg leben viele Obdachlose offen sichtbar in den Zentren der Stadt (zusätzlich zu jenen, in so genannter verdeckter Wohnungslosigkeit). Ihre Präsenz im Herzen von wohlhabenden Einkaufsgegenden wird seit geraumer Zeit kontrovers diskutiert. Die Frage danach, wie diesen Menschen geholfen werden kann, steht jedoch schon längst nicht mehr im Fokus der öffentlichen Diskussionen. Vielmehr geht es im Wesentlichen nur noch um die Verminderung des Geschäftsprofits, die mit den Erscheinungen des Elends verbunden werden. Vertreter/innen von Politik und Gewerbe haben unzählige Versuche gestartet, die marginalisierten Menschen an den Rand der Stadt zu bewegen, exakt dorthin, wo die oben beschriebenen »Nachbarschafts-Aktivisten « auf ihre Weise versuchen, die Sesshaftigkeit dieser unerwünschten Personen zu verhindern.

Obdachlosigkeit und Drogenkonsum sind ebenfalls ein großes Thema für (nicht obdachlose) Ortsansässige, wenn es darum geht, dass ein soziales Projekt versucht, einen Standort für seine Aktivitäten zu finden. Sobald das Wort die Runde macht, ein Projekt suche in einer bestimmten Gegend nach Räumen, dauert es nicht lange, bevor die gut situierten Bewohner/innen ihren Protest offen kundtun und kurzerhand Demonstrationen, Versammlungen etc. organisieren. Ihr Protest gründet sich auf der Annahme, dass mit der Einrichtung eines sozialen Projektes zusätzliche Probleme in ihren Stadtteil verschoben würden, z.B. dass man ihre Kinder durch die Konfrontation mit Konsument/innen illegaler Drogen einer neuen Bedrohung aussetzten würde. Nach erfolgreicher Verhinderung des Zuzugs eines derartigen Projektes, lösen sich die Initiativen zumeist auf.

\section{Jüngste Entwicklungen: Der erfolgreiche Transfer privaten Protestes in politische Programme}

Innerhalb der letzten Jahre ist indessen eine neue Entwicklung zu beobachten. Neu bzw. anders in dem Sinn, dass die öffentlich artikulierte Besorg- nis über die Anzeichen von Armut, die als Störung empfunden werden, erfolgreich in politische Programme transferiert wurde.

Die Hamburger Wahlen von 2001 wurden, wie so viele Wahlen vorher, anhand der Frage entschieden, wer am härtesten gegen Kriminalität vorgehen könne. Die Inszenierung einer »Kriminalitätsbedrohung « im Wahlkampf scheint die Wähler/innen vergessen zu lassen, dass es durchaus auch andere Themen gibt, die zentral für die Politik sein könnten. Kurz bevor die Sozialdemokraten (SPD) und ihre Koalitionspartei (GAL) die Wahlen verloren, versuchten sie damals noch einen letzten Beweis ihrer Härte (am Beispiel der offenen Drogenszenen) zu erbringen, in dem sie den so genannten »Brechmitteleinsatz« erlaubten (die zwangsweise Verabreichung einer Flüssigkeit, die bei der betroffenen Person zum Erbrechen von verschluckten Drogen führen soll). Diese Maßnahme ist in anderen deutschen Städten bereits seit einigen Jahren alltägliche Praxis, wurde in Hamburg jedoch von SPD und GAL immer abgelehnt. Trotz dieser späten Umkehr verloren sie die Wahlen. Die neue Regierung, bestehend aus den Konservativen (CDU), den Liberalen (FDP) und einer neuen Partei, der Partei Rechtsstaatlicher Offensive, führte die Brechmitteleinsätze in aggressiver Weise fort. Dies blieb jedoch nicht ohne Konsequenzen.

Am 9. Dezember 2001 wurde der 19 Jahre alte Achidi J., ein Flüchtling aus Nigeria, wegen des Verdachts auf unerlaubten Handel mit Betäubungsmitteln verhaftet. Er wurde zum »Rechtsmedizinischen Institut der Universitätsklinik Eppendorf « gebracht, wo man ihn dazu bringen sollte, die verschluckten Drogen wieder auszuspucken. Achidi wehrte sich mit allen Kräften gegen die Verabreichung des Brechmittels. Nach mehreren Versuchen wurde ihm die Flüssigkeit über eine Nasensonde verabreicht. Achidi krampfte und verblieb regungslos am Boden, was ihm zunächst als Simulation ausgelegt wurde. Als endlich eine Reanimation in die Wege geleitet wurde, war es bereits zu spät. Achidi verblieb im Koma, bis er am 12. Dezember 2001 an den Folgen dieses Einsatzes starb.

Während die lokalen Regierungsvertreter, ebenso wie die ehemaligen Vertreter der SPD, die Brechmitteleinsätze trotz des »tragischen Ereignisses « als grundsätzlich sinnvolle Verfahrensweise verteidigten, hagelte es Kritik von allen Seiten. Ärzte sahen ihre Profession in einen Handlangerjob für die Ordnungspolitik pervertiert, in der Folter und Todesstrafe an unerwünschten Personen ausgeübt würde. Bürgerrechtsgruppen und sogar die Bischöfin Jepsen, Vorsitzende der Kirchenleitung der Nordelbischen EvangelischLutherischen Kirche, forderten mindestens eine Aussetzung der Verfahrensweise bis geklärt sei, was konkret den Tod von Achidi verursacht hatte. Ein solches Memorandum wurde von den politischen Stellen jedoch abgelehnt. Dagegen ver- öffentliche die Hamburger Presse unzählige Leser/innenbriefe, in denen sich darüber entrüstet wurde, dass die evangelische Kirche einen »schwarzen Drogenhändler « verteidigen würde (Hamburger Abendblatt 14.12.2001, S. 19). In den Augen vieler (auch einiger Presseorgane), war nicht Achidi das Opfer einer menschenverachtenden Verfahrensweise, sondern vielmehr die Personen, die von ihm Drogen gekauft hatten. Er hatte sich entschieden Drogen zu verkaufen, also verdiente er den Tod - so ein führender Presse-Tenor dieser Tage. Menschlichkeit nicht für Dealer - war auch die Maxime der Politik. Die Untersuchung des Falles wurden bald eingestellt und stattdessen wurden zusätzliche Mittel in Höhe von 50.000 Euro für den Ausbau der Brechmitteleinsatzräume bewilligt.

\section{Was ist zu fürchten?}

Die Furcht vor dem Unbekannten, dem Fremden, dem nicht bekannten Lebensstil - diese Ängste scheinen mittlerweile bereitwillig akzeptiert zu werden. Die Feindbilder als Ursachen der Unsicherheit werden quer durch alle politischen Lager hindurch aufgegriffen, egal ob die Parteien zuvor eine ganz andere Linie vertreten haben. Diese ehemalige Sichtweise wurde den Forderungen der privat organisierten Proteste untergeordnet und in politische Programme überführt.

Neben dem oben beschriebenen, gibt es weitere Beispiele der Verquickung von staatlichen und privaten Aktivitäten in diesem Bereich, so z.B. die so genannten »Sicherheitskonferenzen«. Sie wurden von den Parteien (in Hamburg insbesondere der GAL) angeregt, um die Bürgerbeteiligung in einzelnen Stadtteilen zu stärken. Die grundlegende Zielsetzung war, dass aktive Bürger/innen und Institutionen vor Ort sämtliche Themen, Probleme etc. an einen Tisch bringen und durch die gemeinschaftliche Beratung Lösungen finden bzw. in die Wege leiten. Da aber die Finanzierung solch einer Koordinationsrunde und den daraus resultierenden Projekten schwierig war, entschied man sich, den Fokus auf das Thema der öffentlichen Sicherheit zu legen, da hierüber am ehesten eine Finanzierung sichergestellt werden konnte. So wurde von einer Runde bei der es um alle denkbaren Belange einer Gemeinde gehen sollte, eine Runde, die Projekte zur Herstellung öffentlicher Sicherheit thematisierte und initiierte. Wie eine sich selbst erfüllende Prophezeiung werden durch diese Vorgehensweise überhaupt erst Probleme produziert. Sicherheit wird zum Thema, Projekte werden initiiert und daraus wird von Seiten der Bürger/innen wiederum auf eine fehlende Sicherheit geschlossen. Unsicherheit wird so propagiert, bis sie entsteht - in der Rezeption der Menschen, die sich nun überhaupt erst unsicher fühlen. Menschen, die sich ängstigen, sehen nun überall Gefahren und das Klima von Angst produziert wiederum eine angespannte Atmosphäre, die 
sich im schlimmsten Fall in Konflikten und Gewalt entladen kann, damit wird also erst produziert, was vermieden werden sollte - bzw. was nur als finanzierungskräftiger Aufhänger gedacht war.

Ein anderes Beispiel ist die so genannte »Müllpolizei«, die in Hamburg am 1. Januar 2003 ihre Arbeit aufgenommen hat. Sie besteht aus einer Einsatztruppe des Ordnungsamtes und ist inzwischen mit weiterführenden Befugnissen und Aufgaben ausgestattet worden. Anstelle nur noch auf unangeleinte Hunde aufmerksam zu machen und Strafgelder für Blumenkübel über öffentlichem Grund und Boden zu kassieren, darf die Truppe mittlerweile in Zivil Jagd auf Sünder gegen die öffentliche Ästhetik machen, z.B. in Gestalt von Obdachlosen. Ihre Aufgaben gleichen denen, wie sie die Initiativen in Graz oder Rahlstedt auf privater Ebene durchführen. Sie sollen dafür sorgen, dass sich niemand im öffentlichem Raum ungebührlich verhält, dass keine Zigarettenkippe unerlaubt auf den Boden geworfen wird, Graffiti oder marginalisierte Gruppen öffentlich trinken, betteln oder illegale Drogen konsumieren. Diesen »ungebührlichen « Verhaltenweisen unterstellt man den Verlust eines Normbewusstseins, zudem erkennt man sie unhinterfragt als Ursache für die Störung des subjektiven Sicherheitsgefühls der Masse an.

Diese Tendenzen sind aber nicht nur Hamburger Phänomene. Vielmehr ist Hamburg eher einer der letzten Orte, an denen dieser Trend umgesetzt wird. Oberflächlich betrachtet sieht es aus, als ob niemand in all den Jahren des Bestehens offener Drogenszenen und Obdachlosigkeit in deutschen Großstädten in der Lage war, mit zufriedenstellenden Lösungen aufwarten zu können. Eine Weile suchte man nach Konzepten, die allen Seiten nutzen würden. Konzepte, die den Menschen helfen würden, die in Not sind und die gleichwohl dafür Sorge tragen würden, dass sich die Gewerbetreibenden (u.Ä.) nicht mehr mit derartigen öffentlichen Ärgernissen auseinander zusetzen hätten. Es sieht so aus, als ob die Regierungen Hamburgs ebenso wie ihre Wähler/innen, die Geduld mit der Suche nach Lösungen inzwischen ganz aufgegeben haben und sich nur noch mit der Variation von »Säuberungsmaßnahmen« ihrer Stadtzentren beschäftigen würden.

Vielleicht hat aber auch einfach ein grundlegender Einstellungswechsel stattgefunden - ein Zeichen der neoliberalen Zeiten, die eine zunehmende Indifferenz gegenüber den sozialen Gegenwarts-Problemen mit sich bringt. Dies würde erklären, warum es keine moralischen Entrüstungsstürme mehr gibt, wenn ein Konsumraum seine Pforten öffnet oder Heroinvergabeprogramme ihre Arbeit aufnehmen. Diese Konzepte werden mit erheblichen Vorteilen für die öffentliche Ästhetik verbunden, da sie marginalisierte Gruppen mitsamt ihrem Konsum in geschlossene Räume verbannen und damit ihrer Sichtbarkeit in der Öffentlichkeit entziehen. Auf diese Weise entsprechen sie genau den Interessen jener, die sich am personifizierten Antlitz von Deprivation stören. Dies wird seit einigen Jahren nun schon in Großstädten wie Frankfurt oder Hamburg praktiziert.

Andererseits kann man einwenden, dass es ebenso viel Engagement von Menschen gibt, die sich für marginalisierte Gruppen einsetzen, wie z.B. für Obdachlose, Konsumenten illegaler Drogen etc. Auch zum Thema des Brechmitteleinsatzes gab es diverse Initiativen, die Demonstrationen organisierten und versuchten, finanzielle Mittel einzuwerben, um die Verantwortlichen für Achidis Tod rechtlich belangen zu können.

Vielleicht sollte die Frage daher sein, ob es andere Wege gibt, differenziertere Ansichten zu Phänomenen zu transportieren, die mit Angst besetzt sind. »Die Öffentlichkeit « besteht aus vielen verschiedenen Personen, deren Rezeption der Nachrichten ebenso unterschiedlich sind. Von der Einseitigkeit der medialen Berichterstattung auf die Rezeption durch das Publikum zu schließen ist daher kurzsichtig. Und genau dies sollte Mut machen, es verstärkt $\mathrm{zu}$ versuchen, auch komplexere Erklärungen von gesellschaftlichen Problemen zu vermitteln. Dies impliziert eine Herausforderung der dominierenden Feindbilder, die die Obdachlosen, die Konsument/innen illegaler Drogen im öffentlichen Raum und die Drogenhändler/innen beinhalten. Ihre Darstellung korrespondiert mit vielen Ängsten (Sozialer Abbau, Jugend, Rebellion, Unverdienter Genuss, das Unbekannte / Fremde etc.) und weil die Menschen so wenig über sie wissen, können sie leicht als Sündenböcke benutzt werden. Das heißt, ihr Image wird für diverse andere Themen genutzt, nur um von grundlegenden sozialen Problemen abzulenken, und um einfache Lösungen für komplexe Probleme zu präsentieren.

"We should not overlook that, perhaps in most ways, even those who are very different are much like the rest of us after all" (Blum, R.: »The Dream Seller «, San Francisco 1972, S. 121). Diese resümierenden Worte von Richard Blum anlässlich einer Interviewstudie im Drogenhandel, können ein gutes gedankliches Training sein, um Vorurteile abzubauen. Vorurteile, die vorhanden sind, wenn man sich mit Personen befasst, die illegale Drogen konsumieren oder mit ihnen handeln. Ebenso aber auch solche, die beinahe alles umgibt, was uns fremd erscheint, seltsam - unbekannt und daher furchteinflössend wirkt. Der Verwendung dieser Personen zur einfachen Bedrohungsinszenierung könnte so der Boden entzogen werden.

Bettina Paul ist Mitarbeiterin am Institut für Kriminologische Sozialforschung und am Aufbau- und Kontaktstudium Kriminologie, Fachbereich Sozialwissenschaften der Universität Hamburg

TERMINAL

Empirische Polizeiforschung "Innen- und Außenansichten der Polizei«

8.-10. Juli 2004

Frankfurt am Main,

in den Räumen des Hessischen

Landessportbundes

(Nahe Waldstadion/Flughafen)

hermann.gross@vfh-hessen.de

Internationaler Studientag "Stalking-Möglichkeiten und Grenzen der Intervention«

26. März 2004

an der Universität Hamburg,

Institut für Kriminologische

Sozialforschung

astksek@uni-hamburg.de

Programm:

Internationale Experten der Stalking-Forschung sprechen u.a. über

- die auswirkungen der Taten auf die Opfer

- Fallanalyse und-management

- den Zusammenhang zwischen Drohungen und Gewalt

- das erjöhte Viktimisierungsrisiko von Ärzten und Therapeuten

Zielgruppe:

alle Berufsgruppen, die mit Opfern wiederholter Belästigung/Belästigung zu tun haben und Wissenschaftler mit entsprechenden thematischen Schwerpunkten

\section{Deutscher Jugendgerichtstag Verantwortung für Jugend} Qualitätssicherung und Perspektiven in der Jugendkriminalrechtspflege DVJ] 25.-28. September 2004 in Leipzig www.dvjj.de 